\title{
Population control under various family planning schemes in Uttar Pradesh, India
}

\author{
Prashant Verma*, Kaushalendra Kumar Singh, Anjali Singh and Ujjaval Srivastava
}

\author{
* Correspondence: \\ prashantvermag@gmail.com \\ Department of Statistics, Banaras \\ Hindu University, Varanasi 221005, \\ India
}

\begin{abstract}
Contraceptive policies have been derived to achieve desired reductions in the growth rate and also applied to the data of Uttar Pradesh, India, for illustration. Using the Lotka integral equation for stable population, expressions for the proportion of contraceptive users at different ages have been obtained. In order to reduce the present annual growth rate from 0.036 to 0.020 , it is imperative that there will be $42 \%$ contraceptive users at age 20 , assuming that $40 \%$ of them will discontinue at age 25 and 30\% will resume the contraceptive use at age 30. Assuming furthermore that $75 \%$ of females start using contraceptives at the age of 23 years, $50 \%$ of the remaining females start using them at the age of 28 years, and the rest of the females start using them at the age of 32 years, if we set the minimum age at marriage at 20 years, a reduction of 0.019 in the growth rate will be obtained. This study describes how the level of contraceptive use at different age groups of females has helped reduce the growth rate in the state of Uttar Pradesh. It also promotes delayed marriage in the region.
\end{abstract}

\section{Introduction}

India, a country situated in South Asia, is the second most populous country on earth, with $16.7 \%$ of the planet's inhabitants. Population growth in India has been an issue of attention among researchers and policymakers for decades. In Kulkarni and Alagarajan (2005), they addressed the issue of religious differentials in the population growth in India and contraceptive practices. As per the vision of the "National Population Policy (2000)," a target total fertility rate (TFR) of 2.1 was set to be obtained by 2010. In spite of a noteworthy decline in the TFR from 3.6 in 1991 to 2.4 in 2012 (Sample Registration System (SRS) 2012), India is yet to achieve the replacement level of 2.1. By 2012, more than 20 states/union territories of India had reached the replacement level of TFR. Uttar Pradesh (UP), the state with the largest population, still had a high TFR (3.3) (SRS 2012). Uttar Pradesh, accommodating approximately 204 million people, has a high level of fertility and contributes significantly to the population growth of India. Due to the above fact, several works have been done regarding the population growth, fertility transition, and contraceptive behaviors in Uttar Pradesh by many researchers: Elder (1974), Stephenson et al. (2006), and Das and Mohanty (2012). 
According to Srinivasan (1977), "The gaps between the target fixed and the accomplishment in the earlier five-year plan terms may be due to the deficiencies in the implementation of the plan by the government or due to flaws in the setting up of the targets for several methods of family planning or due to errors in the assumptions relating to the possibilities of achieving desired national goals within a specified period of time."

Looking at the current scenario (NFHS-4, 2015-16), India shows that the TFR for India is 2.2, while for UP, it is 2.7. In NFHS-3 (2005-06), UP's TFR was 3.8. This is an excellent progress in 10 years, and a steady progress over previous decades; however, the average number of living children in UP is three to the married women who have at least one child (NFHS-4). Similarly, the median age at marriage, which is another slow moving variable, has improved for UP. In 20052006, according to the NFHS, the median age of women at first marriage in UP was 16 years, which has gone up to 19 years as per NFHS-4. The proportion of women marrying before the legal minimum age has also declined sharply. In 2005-2006, about 59\% of women were married before age 18. By 2015-2016, this number had declined to $21 \%$. This proportion is a little higher in rural UP (25\%). Though this is no mean achievement, a further reduction in the TFR and a hike in the age at marriage can contribute to reduce the population growth rate more efficiently. According to the NFHS-4, the total unmet need for family planning has been found to be $18 \%$ in UP, and this is $2 \%$ higher in rural UP (20\%). This shows the need for an efficient strategy to promote contraceptive use among married women in UP, India.

Statistical models, framed within the limits of specific scenarios and assumptions, can usefully project a program's objectives and achievements (Potter and Parker 1964; Singh 1964). A precise theoretical analysis of the effects of sterilization/emigration on population growth has been given by Keyfitz $(1971,1975)$. Keyfitz's results are the origin point for any extensive study of the relationship between population growth and contraceptive devices. Keyfitz (1971) derived several expressions to obtain the proportion of sterilization/emigration at particular age levels to accomplish the desired rate of population growth, taking the baseline population to be stable. Keyfitz $(1971,1975)$ derived some expressions regarding contraceptive policies to investigate the proportions of contraceptive users at different age levels, based on the desired population growth.

Singh and Yadava (1979) have proposed two types of contraceptive policies. The first type of policies attempts to determine the required proportions of contraceptive users to achieve the desired growth rate; conversely, the second type of policies evaluates the reduction in growth rate of population for the assumed proportion of contraceptive users. In this paper, in addition to the first type of policies, we have derived a new contraceptive policy in order to get the desired growth rate and applied it to the data of Uttar Pradesh, India (SRS 2012 and NFHS-4 2015-16) for illustration. Also, the consequences of these policies on population growth have been evaluated. The study population (Uttar Pradesh, India) justifies the assumption of a stable population, since the birth and death rates in Uttar Pradesh have remained almost constant for several years (SRS, 2009-2012). 


\section{Materials and methods}

\section{Contraceptive policies}

Proportions of contraceptive users under different policies to obtain the desired rate of population growth

Singh and Yadava (1979) proposed some hypothetical contraceptive policies (policies I-III), considering the various proportions (as parameters) of women who start using contraceptives and of those who discontinue them.

Keyfitz (1975) observed that a cautious use of a contraceptive policy can result in a sharp decline in the population growth.

The growth rate of the population is determined by fertility and mortality behaviors, assuming the population is closed for migration. This can be explained by the Lotka integral equation, which is as follows:

$$
\int_{\alpha}^{\beta} \exp (-r a) p(a) m(a) d a=1
$$

Where $p(a)$ is the proportion of the females in the population that survives to age $a$, $m(a)$ is the probability that a female of age $a$ will produce a female child in the next $d a$ period of her reproductive cycle, and $\alpha$ and $\beta$ are the lower and upper limits of the reproductive period.

If we want to achieve the desired rate of growth $r^{*}$ from the current pace of growth $r$, then the following contraceptive policies can be worked out:

Policy I: A continued stream of contraceptive users at a single age level $x$ for $x<\beta$. This policy suggests the proportion of women who should start using contraceptives at a certain age and keep using them throughout their reproductive span.

Policy II: A definite proportion $f$ of the study population starts using a protective device (contraceptive) at a particular age level $x$, and a certain proportion $\sigma$ discontinues its use on reaching age $y(x<y<\beta)$.

Policy III: A continued stream of contraceptive users at two age levels $x$ and $y$ independently. This policy suggests two proportions of women who should start using contraceptives at two different ages and use them throughout their reproductive span.

In continuation of the above strategies suggested by Singh and Yadava (1979), we have proposed the following contraceptive policy-policy IV-since the above three policies do not fit the current scenario of the UP state of India and show very less applicability due to their unrealistic assumptions for the contraceptive needs of the region.

Policy IV: A certain proportion $f$ of the population starts to use a contraceptive device at a certain age level $x$, a fixed proportion $\sigma$ discontinues its use on reaching age $y(x<y<\beta)$, and a definite proportion $\lambda$ resumes the use of the contraceptives at age $z(y<z<\beta)$.

Policy IV says that a fixed proportion of women should start using contraceptives after marriage, women who desire a child should discontinue, and after achieving the desired parity, those women should again start using the contraceptives. This policy addresses the current need of the region due to its high applicability and feasibility.

Illustrations for the proportions of contraceptive users as per these policies are given in Appendix 1 and explained by Eqs. (3), (6), (9), and (12), respectively. 
Reduction in the growth rate of population for a given proportion of contraceptive users

In contrast to the above four policies which evaluate the required proportion of contraceptive users for a certain drop in growth rate, Singh and Yadava (1979) suggested policies V and VI, which evaluate the decline in growth rate for certain proportions of contraceptive users at different ages.

The decline in the rate of growth $r$ is evaluated under the following policies (Singh and Yadava 1979):

Policy V: A certain proportion $f$ of the population uses a contraceptive method continuously after reaching age $x(x<\beta)$, and a certain proportion $\sigma$ of the remaining females starts using it at age $y(y>x)$. The remaining population starts using contraceptives at age $z(z>y>x)$.

Policy VI: In addition to policy V, the age at marriage is increased to $k$ for $k>\alpha$.

Under policies V and VI, expressions for the reduction in the rate of growth $r$ are obtained in Appendix 1 and are presented by Eqs. (15) and (18) respectively.

The different population control policies for Uttar Pradesh described above are illustrated with the help of the demographic data (SRS 2012 and NFHS-4).

The contraceptive policies discussed above focus only on the married women of UP (aged 15-49 years), since in India, births and pregnancy are usually reported only after marriage (a data constraint). Given that the modern contraceptive methods have a very low failure rate compared to the classical methods, it is recommended that the medical service providers suggest modern contraceptive methods for population control.

The different values of net maternity function $p(a) m(a)$ corresponding to different ages are required to illustrate the different contraceptive policies mentioned above. The values of $m(a)$ are computed with the help of the SRS (2012) data, and the computational method is given in Appendix 3. The values of $p(a)$ are chosen from South model level 19 (Coale and Demeny 1966). We chose this level due to the similarity in mortality conditions between the region of study and the South model, level 19, in the regional model life table. We have used the same data to compute the value of the rate of growth $r$ and the other population parameters. The complete computational procedure is given in Appendix 2. Tables 1 and 2 present the values of $p(a) m(a)$, the net maternity function; $\mu$, the mean length of generation time; and $R_{0}$, the net reproduction rate, along with other population parameters.

Table 1 Values of $R_{0}, \mu, r$, and net maternity function $p(a) m(a)$ along with the computation of exp $\left(-r^{*}\right.$ a) $p(a) m(a)$ for predicting the proportion of contraceptive users to attain the rate of growth $r^{*}=0.020$ from $r=0.036$

\begin{tabular}{lcccc}
\hline Age $(z)$ & $p(a)$ & $p(a) m(a)$ & $\exp \left[-.02^{*}(z+2.5)\right] p(a) m(a)$ & $\int \exp \left(-r^{*} a\right) p(a) m(a) d a$ \\
\hline 15 & 0.88592 & 0.67148 & 0.47318 & 1.50550 \\
20 & 0.88076 & 0.81029 & 0.51666 & 1.03232 \\
25 & 0.87374 & 0.55575 & 0.32064 & 0.51565 \\
30 & 0.86562 & 0.23103 & 0.12061 & 0.19501 \\
35 & 0.85641 & 0.10658 & 0.05035 & 0.07441 \\
40 & 0.84569 & 0.04522 & 0.01933 & 0.02406 \\
45 & 0.83210 & 0.01224 & 0.00473 & 0.00473
\end{tabular}

$R_{0}=2.43, \mu=24.37, r=0.036$

${ }^{1}$ Here, $z$ is the lower limit of the age class, e.g., in class (15-20), 15 is the lower limit and $a$ is the mid value of age class, e.g., in class $(15-20),(15+2.5)=17.5$ is the age of female used for computing the proportions. Since calculated $m(a)$ in Table 4 is based on the age interval as (15-19), (20-24)...(45-49), $a$ is considered to be the mid value of age interval 
Table 2 Values of $R_{0}, \mu, r$, and net maternity function $p(a) m(a)$ along with the computation of exp $\left(-r^{*}\right.$ a) $p(a) m(a)$ for predicting the proportion of contraceptive users to attain the rate of growth $r^{*}=0.015$ from $r=0.036$

\begin{tabular}{lcccc}
\hline Age $(z)$ & $p(a)$ & $p(a) m(a)$ & $\exp \left[-.015^{*}(z+2.5)\right] p(a) m(a)$ & $\int \exp \left(-r^{*} a\right) p(a) m(a) d a$ \\
\hline 15 & 0.88592 & 0.67148 & 0.51645 & 1.69506 \\
20 & 0.88076 & 0.81029 & 0.57818 & 1.17861 \\
25 & 0.87374 & 0.55575 & 0.36790 & 0.60042 \\
30 & 0.86562 & 0.23103 & 0.14189 & 0.23252 \\
35 & 0.85641 & 0.10658 & 0.06073 & 0.09064 \\
40 & 0.84569 & 0.04522 & 0.02390 & 0.02991 \\
45 & 0.83210 & 0.01224 & 0.00600 & 0.00600 \\
\hline
\end{tabular}

$R_{0}=2.43, \mu=24.37, r=0.036$

${ }^{1}$ Here, $z$ is the lower limit of the age class, e.g., in class (15-20), 15 is the lower limit and $a$ is the mid value of age class, e.g., in class $(15-20),(15+2.5)=17.5$ is the age of female used for computing the proportions. Since calculated $m(a)$ in Table 4 is based on the age interval as (15-19), (20-24)...(45-49), $a$ is considered to be the mid value of age interval

Example: The proportion of females proposed for contraceptive usage at age 20 to bring the present rate of growth 0.036 to 0.020 , as per Eq. (3), is:

$$
\frac{1.50550-1}{1.03232}=0.48967
$$

Example: The proportion of females proposed for contraceptive usage at age 20 to bring the present rate of growth 0.036 to 0.015 , as per Eq. (3), is:

$$
\frac{1.69506-1}{1.17861}=0.58972 \text {. }
$$

\section{Illustrations and conclusions}

\section{Assessment of contraceptive policies (I-IV)}

Assessment of the first four policies has been done for two different desired declines in growth rate.

\section{Assessment of the policies for the target growth rate of 0.020 compared to the current growth rate 0.036}

Let us assume a target annual growth rate for Uttar Pradesh of 0.02 compared to the current growth rate 0.036 per year. The values of $\exp \left(-r^{*} a\right) p(a) m(a)$ for $r^{*}=0.02$ are presented in Table 1. Evaluating Eq. (3) for policy I and the data (Table 1), we found that if females start using contraceptives continuously after age 20 , about $49 \%$ of contraceptive users of the same age will be required to decrease the population growth rate from 0.036 to 0.02 . Similarly, at age 25 years, about $98 \%$ contraceptive users are necessary for the same reduction in the rate of growth in the region if females start using contraceptives continuously after reaching age 25 .

If we take $x=20, y=25$, and $\sigma=0.25$, then for policy II, from Eq. (6), it can be shown that about $61 \%$ of the females at 20 years of age need to adopt contraceptive devices in order to have the rate of growth decline from 0.036 to 0.02 , assuming that $25 \%$ of the users would discontinue at the age of 25 years. If $x=20, y=30$, and $\sigma=0.25$, about $54 \%$ of females are needed to start contraceptive methods at the age of 20 years to get a rate of growth of 0.02 , assuming that $25 \%$ of the users would discontinue at the age of 30 years. 
Under policy III, if $x=20, y=25$, and $f_{2}=0.20$, then from Eq. (9), it is found that it is imperative for about $43 \%$ of females to start using a contraceptive device at the age of 20 years to have a rate of growth of 0.02 per year, assuming that $20 \%$ of the females would adopt contraceptives at the age of 25 years.

Considering the proposed policy IV, if $x=20, y=25, z=30, \sigma=0.40$, and $\lambda=0.30$, then from Eq. (12), we can comment that of the women aged 20 years, $42 \%$ are required to be contraceptive users to reduce the present growth rate from 0.036 to 0.02 , assuming that $40 \%$ of them will discontinue the use of contraceptives at the age of 25 years and 30\% will resume the contraceptive use at age 30 years. Table 3 and Fig. 1 present a snapshot of policy I to policy IV.

\section{Assessment of the policies for the target growth rate of 0.015 compared to the current growth rate 0.036}

Let us assume a desired annual growth rate of 0.015 for Uttar Pradesh compared to the current annual growth rate of 0.036 . The values of $\exp \left(-r^{*} a\right) p(a) m(a)$ for $r^{*}=0.015$ are calculated in Table 2. Evaluating Eq. (3) for policy I and the data (Table 2), we observe that if females start using contraceptives continuously after reaching age 20, about $59 \%$ of the contraceptive users of that age will be required to bring down the growth rate of Uttar Pradesh from 0.036 to 0.015 .

If we take $x=20, y=25$, and $\sigma=0.25$, then for policy II, from the Eq. (6), it can be shown that about $72 \%$ of the females at 20 years of age need to adopt contraceptive methods in order to have the growth rate fall from 0.036 to 0.015 , assuming that $25 \%$ of the users would discontinue at the age of 25 years. If $x=20, y=30$, and $\sigma=0.25$, about $64 \%$ of females are needed to acquire contraceptive methods at the age of 20 years to get a rate of growth of 0.015 , assuming that $25 \%$ of the users would discontinue at the age of 30 years.

Under policy III, if $x=20, y=25$, and $f_{2}=0.20$, then from Eq. (9) it is found that about $54 \%$ of females are needed to start to use a contraceptive device at the age of 25 years to achieve a growth rate of 0.015 , assuming that $20 \%$ of females would adopt contraceptives at the age of 25 years.

Considering the proposed policy IV, if $x=20, y=25, z=30, \sigma=0.40$, and $\lambda=0.30$, then for Eq. (12), we can comment that among women aged 20 years, $51 \%$ of contraceptive users are imperative to reduce the present growth rate from 0.036 to 0.015 , assuming that $40 \%$ of contraceptive users discontinue the use of contraceptives at the age of 25 and $30 \%$ resume the contraceptive use at the age 30 years.

Table 3 Parameters and required proportion of contraceptive users at age 20

\begin{tabular}{lcccc}
\hline For desired growth rate, $r=0.015$ & & & For desired growth rate, $r=0.020$ & \\
\cline { 1 - 2 } Description of policy & $\begin{array}{c}\text { Required } \\
\text { proportion }(f)\end{array}$ & & Description of policy & $\begin{array}{c}\text { Required } \\
\text { proportion }(f)\end{array}$ \\
\hline Policy I $(x=20)$ & 0.59 & & Policy I $(x=20)$ & 0.49 \\
Policy II $(x=20, y=25, \sigma=0.25)$ & 0.72 & & Policy II $(x=20, y=25, \sigma=.025)$ & 0.61 \\
Policy III $\left(x=20, y=25, f_{2}=0.20\right)$ & 0.54 & & Policy III $\left(x=20, y=25, f_{2}=0.20\right)$ & 0.43 \\
Policy IV $(x=20, y=25, z=30, \sigma=$ & 0.51 & & Policy IV $(x=20, y=25, z=30, \sigma=$ & 0.42 \\
$0.40, \lambda=0.30)$ & & & $0.40, \lambda=0.30)$ & \\
\hline
\end{tabular}




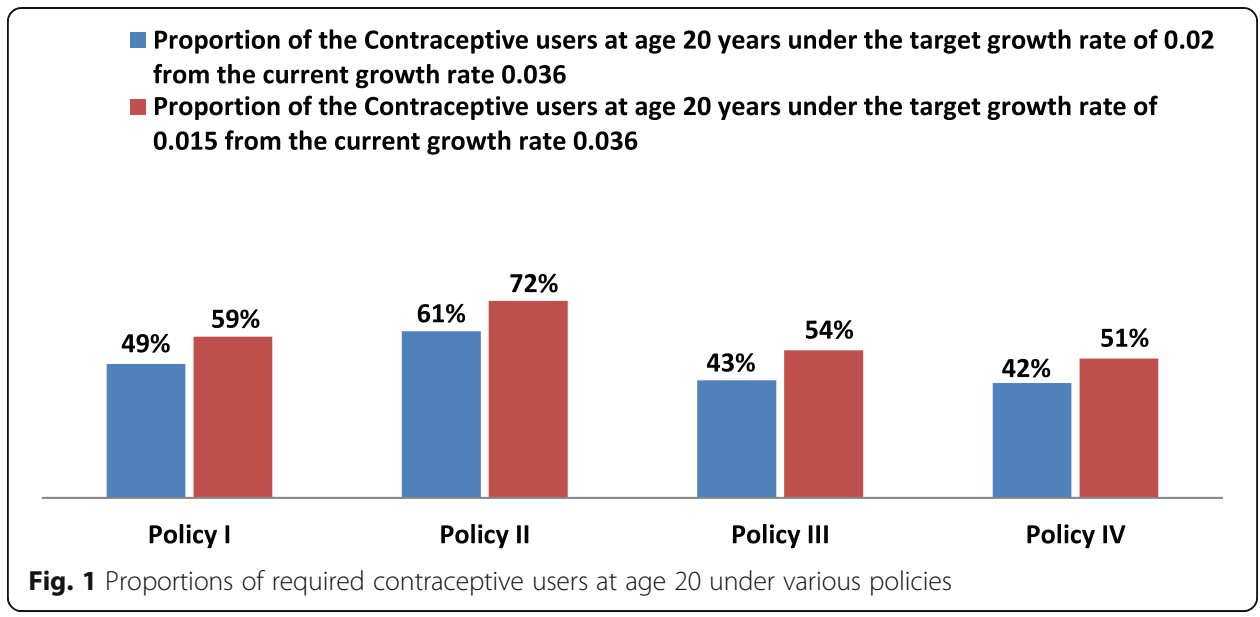

Keeping the above illustrations in mind, it is worthwhile to mention that the proposed policy IV is more feasible and correlates well with the current policies and desire of women to limit their fertility and reach replacement level in Uttar Pradesh, India. Also, the proposed contraceptive policy does not compel women to use contraceptives as a set target, but it suggests policy makers and services providers to encourage couples to use contraceptives, in order to favor women's reproductive rights (as India is a signatory to the Cairo ICPD) and fulfill a desired of fertility replacement in the region.

\section{Assessment of policies $\mathrm{V}$ and $\mathrm{VI}$}

To illustrate policies $\mathrm{V}$ and $\mathrm{VI}$, the values of $f, \sigma, x, y$, and $z$ are required. To make the selections of ages rational, the values of $x, y$, and $z$ are taken approximately as the median ages of women at first, second, and third parity, respectively. Since the data on the median age of females at certain parities was not available in SRS (2012), we computed it from the National Family Health Survey (2015-16) data, with 25, 30, and 34 years, respectively, being the age at first, second, and third parity. Thus, the values of $x, y$, and $z$ have been taken to be 25,30 , and 34 years, respectively. Since the value of $p(a)$ is not available in the model life table corresponding to age 34 years, the value of $\exp \left(-r^{*} a\right)$ $p(a) m(a)$ has been interpolated. The choice of an interpolation formula is largely responsible for the accuracy of the estimates (Scarborough 1955). Gauss' forward interpolation formula has been used to estimate the above expression for females aged 34 years.

Taking $x=25, y=30, z=34, f=0.75$, and $\sigma=0.50$ in Eq. (15), then under policy $\mathrm{V}$, we have the value of $\Delta r$ and the reduction in $r$ as:

$$
\Delta r=0.009, \text { with } R_{0}=1.93
$$

From Eq. (18), under policy VI with $f=0.75, \sigma=0.50, x=25, y=30, z=34$, and $k=20$, we get:

$$
\Delta r=0.021, \text { with } R_{0}=1.44 .
$$

It is worthwhile to mention that the reduction in the rate of growth through policy VI, expressed by Eq. (15), is significantly more than that of policy V represented by Eq. (18). 
This may be due to the fact that fertility between the ages 15 to 20 years is still very high in Uttar Pradesh, India. In India, birth before marriage is not accepted socially. Therefore, a data constraint exists on the fertility before marriage, and it is a convention in most of the large-scale surveys to consider that no births occur before marriage in India. Therefore, in Eq. (17), $m(a)$, the probability of having a female child, is considered to be zero if $a<k$ (age at first marriage) since there is zero probability of birth before marriage as per the data gap. Hence, we can conclude that high fertility in the age group 15-20 years is only due to the low age at marriage in the region. Therefore, increasing the age at marriage along with the use of contraceptives at different ages is a key solution for population control in the region.

\section{Appendix 1}

Keyfitz (1971) has derived that when a proportion $f$ of the population uses any contraceptive device on attaining age $x,(x<\beta$ and $0 \leq f \leq 1)$ the declined rate of growth $r^{*}$ satisfies:

$$
\int_{\alpha}^{\beta} \exp \left(-r^{*} a\right) p(a) m^{\mathrm{I}}(a) d a=1
$$

$$
\text { With, } m^{\mathrm{I}}(a)= \begin{cases}m(a) & \text { if } a<x \\ (1-f) m(a) & \text { if } a \geq x\end{cases}
$$

Solving (2) for $f$,

$$
f=\frac{\int_{\alpha}^{\beta} \exp \left(-r^{*} a\right) p(a) m(a) d a-1}{\int_{x}^{\beta} \exp \left(-r^{*} a\right) p(a) m(a) d a}
$$

Expression (3) can be used to find the proportion of females at age $x$ that would be expected to use the contraceptive device (under policy I) for the desired rate of growth $r^{*}$.

Under policy II, if a specific proportion $f(0 \leq f \leq 1)$ of the population starts using contraceptives, like oral pills, condoms, and IUCD (intrauterine contraceptive device), on reaching age $x$ and a specific proportion $\sigma(\sigma<f)$ discontinues on reaching age $y(x<y<\beta)$, then the declined rate of growth $r^{*}$ fulfills:

$$
\int_{\alpha}^{\beta} \exp \left(-r^{*} a\right) p(a) m^{\mathrm{II}}(a) d a=1
$$

With, $\quad m^{\mathrm{II}}(a)= \begin{cases}m(a) & \text { if } a<x \\ (1-f) m(a) & \text { if } x \leq a<y \\ (1-f+\sigma) m(a) & \text { if } a \geq y\end{cases}$ 


$$
f=\frac{\int_{\alpha}^{\beta} \exp \left(-r^{*} a\right) p(a) m(a) d a+\sigma \int_{y}^{\beta} \exp \left(-r^{*} a\right) p(a) m(a) d a-1}{\int_{x}^{\beta} \exp \left(-r^{*} a\right) p(a) m(a) d a}
$$

Thus for an assumed value of $\sigma$ (discontinuation rate), the value of $f$ can be computed for the desired reduction in the growth rate.

Under policy III, if there exists a continued stream of contraceptive users at two ages, $x$ and $y$, then the reduced growth rate $r^{*}$ satisfies:

$$
\int_{\alpha}^{\beta} \exp \left(-r^{*} a\right) p(a) m^{\mathrm{III}}(a) d a=1
$$

With, $\quad m^{\mathrm{III}}(a)= \begin{cases}m(a) & \text { if } a<x \\ \left(1-f_{1}\right) m(a) & \text { if } x \leq a<y \\ \left(1-f_{1}\right)\left(1-f_{2}\right) m(a) & \text { if } a \geq y\end{cases}$

where $f_{1}$ and $f_{2}$ give the proportions of contraceptive users at ages $x$ and $y$ individually. Solving (7) for (8), we find:

$$
f_{1}=\frac{\int_{\alpha}^{\beta} \exp \left(-r^{*} a\right) p(a) m(a) d a-f_{2} \int_{y}^{\beta} \exp \left(-r^{*} a\right) p(a) m(a) d a-1}{\int_{x}^{\beta} \exp \left(-r^{*} a\right) p(a) m(a) d a-f_{2} \int_{y}^{\beta} \exp \left(-r^{*} a\right) p(a) m(a) d a}
$$

For a given $r^{*}$ and $f_{2}, f_{1}$ can be evaluated with Eq. (9). A similar expression for $f_{2}$ in terms of $f_{1}$ and $r^{*}$ can be worked out.

Considering the proposed policy IV, if a certain proportion of $f(0 \leq f \leq 1)$ of the population starts using contraceptive devices on reaching age $x$, a certain proportion $\sigma(\sigma<f)$ discontinues their use on reaching age $y(x<y<\beta)$, and a certain proportion (who achieved their desired parity) $\lambda(\lambda<\sigma)$ again start using contraceptives at age $z$, then the new rate of growth $r^{*}$ satisfies:

$$
\int_{\alpha}^{\beta} \exp \left(-r^{*} a\right) p(a) m^{\mathrm{IV}}(a) d a=1
$$

With, $\quad m^{\mathrm{IV}}(a)= \begin{cases}m(a) & \text { if } a<x \\ (1-f) m(a) & \text { if } x \leq a<y \\ (1-f+\sigma) m(a) & \text { if } y \leq a<z \\ (1-f+\sigma-\lambda) m(a) & \text { if } a \geq z\end{cases}$ 
$f=\frac{\int_{\alpha}^{\beta} \exp \left(-r^{*} a\right) p(a) m(a) d a+\sigma \int_{y}^{\beta} \exp \left(-r^{*} a\right) p(a) m(a) d a-\lambda \int_{z}^{\beta} \exp \left(-r^{*} a\right) p(a) m(a) d a-1}{\int_{\alpha}^{\beta} \exp \left(-r^{*} a\right) p(a) m(a) d a}$

Under policy $\mathrm{V}$, when a proportion $f$ of the population starts using any contraceptive device continuously on attaining age $x$, a fixed proportion $\sigma$ of the remaining population starts using contraceptives at age $y(y>x)$ and the remaining population starts using contraceptives at age $z(z>y>x)$, the new rate of growth $r$ satisfies:

$$
\int_{\alpha}^{\beta} \exp \left(-r^{\prime} a\right) p(a) m^{\mathrm{V}}(a) d a=1
$$

With, $\quad m^{\mathrm{V}}(a)=\left\{\begin{array}{lr}m(a) & \text { if } a<x \\ (1-f) m(a) & \text { if } x \leq a<y \\ (1-\sigma)(1-f) m(a) & \text { if } y \leq a<z \\ 0 & \text { if } a \geq z\end{array}\right.$

where, $r^{\prime}=r+\Delta_{r}$

$\Delta_{r}$ is the reduction in the growth rate $r$. If $\Delta_{r}$ is small enough that

$$
\exp -\left(\Delta_{r}\right)_{a} \simeq 1-\left(\Delta_{r}\right)_{a}
$$

And also if $f$ and $\sigma$ are small enough that $\left(f \Delta_{r}\right)$ and $(1-f) \Delta_{r}$ are neglected, the expression for $\Delta_{r}$ comes to:

$\Delta_{r} \simeq \frac{f \int_{x}^{z} \exp (-r a) p(a) m(a) d a+\sigma(1-f) \int_{y}^{z} \exp (-r a) p(a) m(a) d a-\int_{\alpha}^{z} \exp (-r a) p(a) m(a) d a+1}{\int_{\alpha}^{z} a \exp (-r a) p(a) m(a) d a}$

It has been advised that an increase in the age at marriage be considered as a technique of controlling the growth of population. If the age at marriage is increased to $k$ years for $k>a$, as a supplement to policy $\mathrm{V}$, then under policy VI, the new rate of growth $r$ satisfies:

$$
\int_{\alpha}^{\beta} \exp \left(-r^{\prime} a\right) p(a) m^{\mathrm{VI}}(a) d a=1
$$


With, $\quad m^{\mathrm{VI}}(a)=\left\{\begin{array}{lr}0 & \text { if } a<k \\ m(a) & \text { if } k \leq a<x \\ (1-f) m(a) & \text { if } x \leq a<y \\ (1-\sigma)(1-f) m(a) & \text { if } y \leq a<z \\ 0 & \text { if } a \geq z\end{array}\right.$

The value of $\Delta_{r}$ can be obtained under policy VI with the fertility schedule (17) as follows:

$$
\Delta_{r} \simeq \frac{f \int_{x}^{z} \exp (-r a) p(a) m(a) d a+\sigma(1-f) \int_{y}^{z} \exp (-r a) p(a) m(a) d a-\int_{k}^{z} \exp (-r a) p(a) m(a) d a+1}{\int_{k}^{z} a \exp (-r a) p(a) m(a) d a}
$$

\section{Appendix 2}

\section{Computational procedure}

The current growth rate of Uttar Pradesh is computed by using the standard formula of stable population (Coale 1972) as:

$$
r=\log R_{0} / T
$$

where $T$ is the mean length of the generation in the stable population. The mean length of the generation or average generation length is the average age of mother of a number of children, either in one generation or across several generations. For example, if a woman had three children when aged 27,30 , and 36 , her average generation length is 31 years $((27+30+36) / 3=31)$.

\section{Appendix 3}

\section{Computation of $m(a)$}

The number of births occurring during a given year or reference period per 1000 women of reproductive age classified in single or five-year age groups is termed as age-specific fertility rates (ASFR). Since the computation of ASFR includes all the women, irrespective of their marital status, Singh and Yadava (1979) multiply ASFR by the proportion of eligible women of the particular age group. Due to the unavailability of data on the proportion of eligible women in SRS (2012), age-specific marital fertility rates (ASMFR) have been used for each age group. Multiplying ASFR by sex ratio and 5 (due to 5 -year age groups), the values of $m(a)$ can be obtained as follows:

$$
m(a)=A S M F R \times Q \times 5
$$

where $Q$ is the sex ratio at birth. The value of $Q$ in Uttar Pradesh, as per SRS (2012) data, is 0.467 female births per birth. The values of $Q$, along with ASMFR, are presented in Table 4. 
Table 4 The values of age-specific marital fertility rates (ASMFR), values of $m(a)$, and $p(a)$ values obtained from model life table for each age group

\begin{tabular}{llll}
\hline Age group & ASMFR & $m(a)$ & $p(a)$ \\
\hline $15-19$ & 0.32460 & 0.75794 & 0.88592 \\
$20-24$ & 0.39400 & 0.91999 & 0.88076 \\
$25-29$ & 0.27240 & 0.63605 & 0.87374 \\
$30-34$ & 0.11430 & 0.26689 & 0.86562 \\
$35-39$ & 0.05330 & 0.12446 & 0.85641 \\
$40-44$ & 0.02290 & 0.05347 & 0.84569 \\
$45-49$ & 0.00630 & 0.01471 & 0.83210 \\
\hline
\end{tabular}

\section{Abbreviations}

ASFR: Age-specific fertility rates; ASMFR: Age-specific marital fertility rates; IUCD: Intrauterine contraceptive device: SRS: Sample Registration System; TFR: Total fertility rate

\section{Funding}

No funding was received to conduct this study.

\section{Availability of data and materials}

The data used for the analysis are available on request.

\section{Authors' contributions}

PV proposed the problem, designed the study, analyzed the data, and did the literature writing. Prof. KKS revised the manuscript critically for important intellectual content and gave the final approval of the version to be published. AS and US reviewed the literature and conceptualize the problem. All authors read and approved the final manuscript.

\section{Competing interests}

The authors declare that they have no competing interests.

\section{Publisher's Note}

Springer Nature remains neutral with regard to jurisdictional claims in published maps and institutional affiliations.

Received: 15 May 2018 Accepted: 27 December 2018

Published online: 08 February 2019

\section{References}

Coale, A. J. (1972). The growth and structure of human population. Princeton, N.J: Princeton University Press.

Coale, A. J., \& Demeny, P. (1966). Regional model life tables and stable population. Princeton, N.J: Princeton University Press.

Das, M., \& Mohanty, S. K. (2012). Spatial pattern of fertility transition in Uttar Pradesh and Bihar: a district level analysis. Genus, 68(2), 81-106.

Elder, R. (1974). Targets versus extension education: the family planning programme in Uttar Pradesh, India. Population Studies, 28(2), 249-261.

Keyfitz, N. (1971). Migration as a means of population control. Population Studies, 25(1), 63-72.

Keyfitz, N. (1975). Reproductive value: with applications to migration, contraception, and zero population growth. In Quantitative sociology (p. 587). San Francisco: Academic Press (eBook - ISBN: 978148328818).

Kulkarni, P., \& Alagarajan, M. (2005). Population growth, fertility, and religion in India. Economic and Political Weekly, 40(5), 403-410

National Family Health Survey-3, India (2005-06). "National Report". http://rchiips.org/nfhs/NFHS-3\%20Data/NOL-1/India_ volume_I_corrected_17oct08.pdf.

National Family Health Survey-4, India (2015-16). "National Report". http://rchiips.org/nfhs/NFHS-4Reports/India.pdf.

National Population Policy (2000), India. https://www.nhp.gov.in/national-population-policy-2000_pg

Potter, R. G., \& Parker, M. P. (1964). Population studies (London) 18(1), 99-116.

Singh, S. N. (1964). On the time of first birth. Sankhyā: The Indian Journal of Statistics, Series B, 26(1/2), 95-102

Singh, S. N., \& Yadava, K. N. S. (1979). Reduction in population growth under different contraceptive policies. Journal of Biosciences, 1(2), 215-222.

Srinivasan, K. (1977). Impact in measuring the impact of family planning, a short guide (pp. 41-73). Paras: Development Centre of the Organisation for Economic: Cooperation and Development.

SRS (2012), Statistical reports, published by Government of India, Ministry of Home Affairs, Office of the Registrar General \& Census Commissioner, India.

Stephenson, R., Koenig, M., \& Ahmed, S. (2006). Domestic violence and contraceptive adoption in Uttar Pradesh, India. Studies in Family Planning, 37(2), 75-86

Scarborough, J. B. (1955). Numerical Mathematical. Oxford and IBH Publishing. 